\title{
The Double-Slit Experiment and Particle-Wave Duality: Toward a Novel Quantum Interpretation
}

\author{
Itzhak Orion $^{1}$, Michael Laitman ${ }^{2}$ \\ ${ }^{1}$ Ben-Gurion University of the Negev, Beer-Sheva, Israel \\ ${ }^{2}$ Ashlag Research Institute, Ramat-Gan, Israel \\ E-mail: iorion@bgu.ac.il \\ Received March 7, 2010; revised March 29, 2010; accepted April 12, 2010
}

\begin{abstract}
The double-slit experiment demonstrates the quantum physics particle-wave duality problem. Over the last decades many interpretations were introduced to the quantum theory perception problem. In most cases there was use of unclear terms, or obscure processes in these interpretations, such as particle splitting. In this paper we propose a novel concept to explain the experiment based on two postulates: The Equivalence of Form (EoF), and the particles connection to other particles, effectively functioning as a group. These two conditions are necessary to maintain wave qualities in the collective relations, and therefore cannot exist in a single particle. De Broglie introduced the mathematical relation of particle to wave; however, he did not specify the conditions for that. The proposed interpretation is a new way of looking at particles as a united group, the Kevutsa, which has a higher order level of matter. A series of identical particles maintain additional qualities to show a large united, correlated motion that we observe as waves transport through systems.
\end{abstract}

Keywords: Particle-Wave Duality; Interference; Quantum Theory; Electrons Diffraction

\section{Introduction}

\subsection{The Huygens-Fresnel principle}

Wave propagation can be well explained by the Huygens-Fresnel principle, as a whole space filled with a plane wave (at distance for example) advancing into a certain slit. The space is governed by the wave in a tight causality since a wave phenomenon can be described only if it is occupying a group of points or a geometrical place. According to this principle, each point along the wave-front can act as a source of secondary waves.

The Huygens-Fresnel principle is the very method to explain the formation of wave diffraction or interference behind single or double slits.

\subsection{Quantum Interpretations}

The problematic view of the quantum reality compared to the observed macroscopic reality lays on three principles:

1) The quantization of electron energy or levels in the atomic structure.

2) The particle-wave duality.
3) The Heisenberg uncertainty principle.

Although each of these principles is observed, quantum reality is still considered strange, and the interpretations usually define unique terminologies, not in use in other fields of physics. In this paper, we introduce a new interpretation for the main experiment that impresses the particle-wave duality; the double slit electron interference experiment.

The particle-wave duality was first introduced by L. de Broglie: A particle has a wavelength or a frequency due to its momentum (p) or its energy (E), respectively.

$$
\lambda=\frac{h}{p} ; \quad f=\frac{E}{h}
$$

where, $h$ is the Planck's constant.

De Broglie wave's expression was proved in observations of a wave's formation, such as diffraction of particles.

Since the late 1920s, many interpretations of the quantum reality have been suggested, the most famous among them is The Copenhagen interpretation [1] introduced by Niels Bohr and Werner Heisenberg. The Copenhagen interpretation is relaying on the Max Born explanation of the wave functions having an abstract mathematical meaning, enabling us to reveal some statistical quantities 
regarding the particles and their states. In the Copenhagen interpretation, as the "standard" quantum methodology, the particle has various probabilities to be measured in different states, and eventually the measurement is randomly obtained. This point of view opened the possibility of freedom since it holds the meaning of 'unknown intermediate states' which are all real until a measurement "chooses" one of these states to stay real.

Several quantum interpretations proposed that the observer is involved in the experimental results in case of quantum systems: Consciousness causes collapse [2], Participatory Anthropic Principle (PAP) [3], and the Many minds interpretation [4]. In this reality there is no way to separate between the experiment and the observer, which gives an air of "You see what you decide to see" to the whole physical reality. Therefore, there is no physical reality but a psychological reality (or we are asked to stop 'doing physics' from this point forth).

This paper aims to propose a new quantum interpretation by means of a new approach to look at electrons. In the following explanations, time is to be interpreted in terms of a series of changes in a given system, similar to a stationary state where there is no need to assume time-dependent variables.

\section{Electron Double Slits Experiments}

The first electron diffraction phenomenon was observed in an experiment performed by Davisson and Germer on nickel crystals in 1927 [5]. Slits system for the electron double slits experiment was developed later, and Jönsson published his experimental results on 1961 in Zeitschrift für Physik [161, 454 (1961)] (translation was published in 1974 in AJP [6]). R. P. Crease published this experiment under the title "The most beautiful experiment" in Physics World 2002 (Sep. 1) [7]. These physical experiments, which were repeated after the technology improvements show how interesting phenomenon is the double slits experiment.

\section{1. "Gedanken Experiment"}

In quantum physics textbooks, electron beams are described as though they are running in a "Gedanken Experiment”. The best description of that appears in Richard P. Feynman's, The Feynman Lectures on Physics Volume 3 Quantum Mechanics, (Chapter 1) [8]. The description starts with a double-slit wall and an electron gun shooting electrons toward the wall. Behind that wall is a screen that shows the intensity at each point. Instead of a typical distribution of balls on the screen behind, an interference pattern appeared on the screen, similar to waves interference. Analyzing the electrons making this pattern in a "Gedanken Experiment" is done by checking through which slit each electron traveled, assuming that an electron can only travel through one slit at a time. However, if one keeps track by a flashlight where through each electron goes, the interference pattern is not observed anymore. Instead, a simple distribution similar to the case of the balls appears. Shooting electrons one at a time or as a whole fluency does not have any impact on the interference pattern.

The apparent problem of destroying the interference pattern by viewing the electrons through slit that they pass is explained by either of the followings:

1) The Heisenberg uncertainty principle.

2) 'Hidden' variables - inner properties of electron.

Einstein suspected that quantum theory is incomplete, which means that there had to be 'hidden' variables in quantum theory. The EPR (Einstein-Podolsky-Rosen) experiment was carried out in 1937 in order to inspect if quantum theory is a complete physical theory or not [9]. In contrast, John Bell, in his 1964 paper [10], very clearly showed that quantum mechanics and Einstein's assumptions lead to different results; hence the 'hidden' variables assumption was neglected.

The special assumptions of the Copenhagen interpretation contained the following expressions:

- Single electron interferes with itself;

- Single electron goes through both slits;

- Superposition of possibilities with each other.

- The observer made the wave function collapse.

Physics, therefore, limited itself to computing probabilities, and omitted the ability to give common-sense explanations and a natural understanding of the nature of electrons.

\section{The Group (Kevutsa) Interpretation}

Group of electrons and their act as a whole group is the interpretation proposed in this paper. As in the case of a stationary system, a series of actions of electrons of the same group are not separate by a time interval, due to the system keeping its unity connection. The electrons system can assumed to be united, due to the Equivalence of Form (EoF) of elementary particles in the group. It is known that the electrons are completely identical in the double slits experiments, even their energy must be equal in order to produce a fair interference pattern on the screen. The identity of electrons is so high that it was noted by R. Feyman's Nobel lecture in 1965 as "there is a single electron needed in order to describe the whole universe, since it can propagate through space and time in such a way as to appear in many places simultaneously".

In macroscopic subjects, such as marbles or bullets, the Equivalence of Form (EoF) condition is not satisfied, and therefore they cannot act as a group. These bullets are always different from one another. The bullets are not having EoF among their bundle, and therefore will 
not possess the Kevutsa (group) properties.

The Kevutsa interpretation introduces here a new property or properties that must be held by a complete group of particles, connected by EoF, to perform wave actions. The group of particles must be considered a united system that has all the variables at the same time to make a connected motion like a wave-form of light. The wave quality of the connected group leads it to obey the Huygens-Fresnel principle, which belongs only to waves. In the Kevutsa interpretation there are no 'hidden' variables in each electron of the experiment. Instead, the connection between electrons gives more degrees of freedom by which additional variables can be related.

The mathematical meaning of the connection between electrons can be illustrated if one defines three dimensional coordinates as the state values of a separated electron defined in a 3 electrons system:

$$
a_{1}\left(x_{1}, y_{1}, z_{1}\right) ; a_{2}\left(x_{2}, y_{2}, z_{2}\right) ; a_{3}\left(x_{3}, y_{3}, z_{3}\right)
$$

However adjoined 3 electrons will be defined by:

$$
\begin{aligned}
& a_{1}\left(\left(x_{3}-x_{1}\right),\left(y_{3}-y_{1}\right),\left(z_{3}-z_{1}\right),\left(x_{2}-x_{1}\right),\left(y_{2}-y_{1}\right),\left(z_{2}-z_{1}\right)\right) ; \\
& a_{2}\left(\left(x_{1}-x_{2}\right),\left(y_{1}-y_{2}\right),\left(z_{1}-z_{2}\right),\left(x_{3}-x_{2}\right),\left(y_{3}-y_{2}\right),\left(z_{3}-z_{2}\right)\right) ; \\
& a_{3}\left(\left(x_{1}-x_{3}\right),\left(y_{1}-y_{3}\right),\left(z_{1}-z_{3}\right),\left(x_{2}-x_{3}\right),\left(y_{2}-y_{3}\right),\left(z_{2}-z_{3}\right)\right) .
\end{aligned}
$$

Therefore, the grouping of electrons highly increases the numbers of variables, and each state can hold enough information needed to satisfy the 'hidden' variables problem. The 'hidden variables' in Einstein's assumption were located in each single particle, while here these variables can be occupied in the connection of group of particles.

Measuring each separate electron track using a flashlight is actually an act of separation of electrons out of their united group. A single electron aims to absorb a photon for itself alone, differing it from the group, leads to a lack of the united group description.

The Kevutsa group interpretation point-of-view is different from the Copenhagen interpretation that always strives to predict a single electron destiny, instead of looking at the whole group of non-separated particle system and their results.

De Broglie introduced the mathematical relation between particle properties and its wave properties. How- ever, he did not introduce the conditions by which particles act as a wave. We recognize the EoF principle as a condition for wave formation of particles.

\section{Conclusions}

The Kevutsa interpretation is a new way of looking at particles as a whole, united group, which has a higher level of order of matter. A series of identical particles (with EoF) have more qualities to show a large united (even infinite) correlated motion that we observe as waves transport through systems.

\section{References}

[1] W. Heisenberg, "Physics and Philosophy: The Revolution in Modern Science,” Goerge Allen \& Unwin, London, 1958.

[2] D. Albert, “Quantum Mechanics and Experience," Harvard University Press, Cambridge, 1994, pp. 80-111.

[3] J. D. Barrow and F. J. Tipler, "The Anthropic Cosmological Principle,” Oxford University Press, Oxford, 1986.

[4] H. Everett, "Relative state formulation of quantum mechanics," Reviews of Modern Physics, Vol. 29, 1957, pp. 454-462.

[5] C. Davisson and L. H. Germer, "Reflection of Electrons by a Crystal of Nickel,” Nature, Vol. 119, 1927, pp. 558-560.

[6] C. Jönsson, "Electron Diffraction at Multiple Slits," American Journal of Physics, Vol. 4, 1974, pp. 4-11.

[7] R. P. Crease, “The Most Beautiful Experiment,” Physics World, September 12002.

[8] R. P. Feynman, "The Feynman Lectures on Physics," Addison-Wesley, Vol. 3, 1965, pp. 1-8.

[9] A. Einstein, B. Podolsky and N. Rosen, "Can QuantumMechanical Description of Physical Reality be Considered Complete?” Physical Review, Vol. 47, No. 10, 1935, pp. 777-780.

[10] J. S. Bell, "On the Einstein-Poldolsky-Rosen Paradox,” Physics, Vol. 1, 1964, p. 195. 